\title{
Galaxy Halo Masses from Weak Gravitational Lensing
}

\author{
Rachel Mandelbaum \\ McWilliams Center for Cosmology, Department of Physics, Carnegie Mellon University, \\ Pittsburgh, PA 15213, USA. email: rmandelb@andrew.cmu.edu
}

\begin{abstract}
In this review, I discuss the use of galaxy-galaxy weak lensing measurements to study the masses of dark matter halos in which galaxies reside. After summarizing how weak gravitational lensing measurements can be interpreted in terms of halo mass, I review measurements that were used to derive the relationship between optical galaxy mass tracers, such as stellar mass or luminosity, and dark matter halo mass. Measurements of galaxy-galaxy lensing from the past decade have led to increasingly tight constraints on the connection between dark matter halo mass and optical mass tracers, including both the mean relationships between these quantities and the intrinsic scatter between them. I also review some of the factors that can complicate analysis, such as the choice of modeling procedure, and choices made when dividing up samples of lens galaxies.
\end{abstract}

\section{Introduction}

The field of galaxy formation and evolution seeks to explain the evolutionary history of galaxies, but is handicapped by the difficulties in observing the dark matter field in which the galaxies form and evolve. The connection between galaxies and their host dark matter halos is an essential ingredient in the physics of galaxy formation. One very useful probe of the galaxy-dark matter connection is weak lensing around galaxies, or galaxy-galaxy lensing (e.g., Hoekstra \& Jain 2008).

Gravitational lensing, the deflection of light by mass, induces tangential shear distortions in the shapes of background galaxies around foreground galaxies, allowing direct measurement of the galaxy-matter correlation function. This approach has the advantage of being sensitive to all matter, independent of its dynamical state. The individual distortions are small (typically of order $0.1 \%$ ), but by averaging over all foreground ("lens") galaxies within a given subsample selected based on their properties (a process known as "stacking"), and over all the source galaxies behind them, it is possible to obtain a high signal-to-noise measurement of the shear as a function of angular separation from the galaxy, known as the galaxy-galaxy lensing signal.

In the past $\sim 15$ years, the quantity of imaging data with high-quality galaxy shape information and with some redshift information (either spectroscopic or photometric redshifts) has drastically increased, in large part due to the efforts of major surveys such as the Red-Sequence Cluster Survey (RCS, Gladders \& Yee 2005) and RCS2 (Gilbank et al. 2011), the Sloan Digital Sky Survey (SDSS, York et al. 2000), the Canada-FranceHawaii Telescope Lensing Survey (CFHTLenS, Heymans et al. 2012; Erben et al. 2013), and the Hubble Space Telescope (HST) COSMOS Survey (Koekemoer et al. 2007; Scoville, et al. 2007a; Scoville et al. 2007b). The increase in data quantity and quality, plus the appeal of a measurement method that is directly sensitive to the dark matter, has led to tremendous progress in the use of galaxy-galaxy lensing to study the connection between galaxies and matter. 
The purpose of this review is to describe some of the studies that have analyzed galaxygalaxy weak lensing measurements to learn about the masses of dark matter halos around galaxies, and to highlight important lessons learned and unsolved aspects of this problem that require more work in future, for example for the next generation of large lensing surveys that will measure weak gravitational lensing more precisely than has been done with current datasets. The topic of this review is limited to relatively general studies of typical galaxies, leaving aside studies of particular galaxy types using weak gravitational lensing (e.g., Mandelbaum et al. 2009; Dutton et al. 2010; Reyes et al. 2012), studies of the radial profiles of dark matter halos with weak lensing (e.g., Gavazzi et al. 2007; Mandelbaum et al. 2008; Schulz et al. 2010), and studies of group or galaxy cluster dark matter halos (e.g., Hoekstra et al. 2013).

\section{Theory}

Weak gravitational lensing, the deflection of light rays by the mass in matter along the line-of-sight, results in coherent shape distortions (shears) in the background galaxies. The strength of the lensing shear depends on the mass in the lens object (galaxy or cluster), the separation on the sky between the lens and the source object, and the line-of-sight distances to lens and source.

A galaxy-galaxy weak lensing measurement probes the connection between galaxies and matter via the cross-correlation functions $\xi_{\mathrm{gm}}(\vec{r})$, which can be related to the projected surface density of matter around the lens galaxies

$$
\Sigma(R)=\bar{\rho} \int\left[1+\xi_{\mathrm{gm}}\left(\sqrt{R^{2}+\chi^{2}}\right)\right] d \chi .
$$

where $R$ is the transverse separation and $\chi$ the radial direction over which we are projectingt. The surface density is then related to the observable quantity for lensing, the differential surface density,

$$
\Delta \Sigma(R)=\gamma_{t}(R) \Sigma_{c}=\bar{\Sigma}(<R)-\Sigma(R),
$$

in the weak lensing limit, for a matter distribution that is axisymmetric along the line of sight (which is naturally achieved when stacking thousands of lens galaxies to determine their average lensing signal). Typical galaxy-galaxy lensing measurements require substantial numbers of lens and source galaxies in order to average out the random component of the source galaxy shapes, which is typically the dominant source of noise in the measurement. This observable quantity can be expressed as the product of two factors, a tangential shear $\gamma_{t}$ and a geometric factor

$$
\Sigma_{c}=\frac{c^{2}}{4 \pi G} \frac{D_{S}}{D_{L} D_{L S}}
$$

where $D_{L}$ and $D_{S}$ are angular diameter distances to the lens and source, and $D_{L S}$ is the angular diameter distance between the lens and source.

For isolated galaxies at the center of dark matter halos, the surface density of surrounding matter can be written in terms of the $3 \mathrm{~d}$ density profile $\rho(r)$ on small scales, as

$$
\Sigma(R)=\int_{-\infty}^{\infty} \rho\left(r=\sqrt{\chi^{2}+R^{2}}\right) d \chi .
$$

However, there are additional contributions due to those galaxies that are satellites, for

$\dagger$ We are ignoring the effects from the radial window, which is broad enough that it is not relevant at galaxy scales; see section 2.3 of Guzik \& Seljak (2001) for details. 
which the host dark matter halo leads to a contribution for $R$ in the hundreds of kiloparsec to $1 \mathrm{Mpc}$ range. On even larger scales, there is a small but significant contribution due to the matter in structures that are physically associated with but not inside of the halo in which the lens galaxy resides (the "2-halo term"). As a result, the interpretation of the lensing signal can be complicated, depending on the local environment of the galaxies (mixture of isolated and central galaxies vs. satellites) and the separations $R$ used (larger scales requires modeling of the term due to large-scale structure). Moreover, the stacking process means that the average profile can be affected not just by mean relationships between mass and observables, but also by scatter in those relationships. Different studies have taken different approaches to these interpretation challenges, as I will describe below.

\section{Results}

Among the first galaxy-galaxy lensing results with detailed modeling of the mass distributions of large samples of foreground lens galaxies were Hoekstra et al. (2005), Mandelbaum et al. (2006), and Heymans et al. (2006).

Hoekstra et al. (2005) used data from the RCS to identify isolated galaxies and measure their lensing signals as a function of stellar mass or luminosity. Due to the use of isolated galaxies, their approach was to fit the signals to an NFW (Navarro et al. 1996) profile, ignoring the possibility of contributions from host halos of those galaxies that may be satellites in larger halos, and restricting to small enough scales that the 2-halo term is negligible. They find a relationship between halo mass and luminosity that goes like $M_{\text {halo }} \propto L^{1.5}$. This result is based on the best-fit mass for samples with some average luminosity, ignoring scatter between mass and luminosity. They also made these measurements for early and late-type galaxy samples split based on color, and found $M_{\text {halo }} / M_{*}$ higher by a factor of $\sim 2$ for early types. Dividing $M_{*} / M_{\text {halo }}$ by the cosmological baryon fraction to estimate an efficiency of conversion of baryons to stars,

$$
\eta=\frac{M_{*}}{M_{\text {halo }}} \frac{\Omega_{\mathrm{m}}}{\Omega_{\mathrm{b}}}
$$

they found $\eta \sim 33 \%$ and $\sim 14 \%$, respectively, for early and late type galaxies.

Using data from the SDSS Main spectroscopic galaxy sample, Mandelbaum et al. (2006) analyzed the host halo mass for early and late-type central galaxies as a function of their stellar mass and luminosity, for galaxies at a typical redshift of $\langle z\rangle \sim 0.1$ (lower than the RCS study). In this work, the split into early versus late types was achieved using a morphological estimator (not color). This work also estimated the satellite fractions purely based on the lensing signal alone. In order to interpret the lensing signals in terms of central and satellite galaxies in a statistical sense, this work used a halo model (e.g., Seljak 2000; Cooray \& Sheth 2002) formalism that was tested for these purposes on mock galaxy samples derived from $N$-body simulations by Mandelbaum et al. (2005). The simple halo model that was used had only two free parameters, with the rest fixed to some values selected based on the $N$-body simulation analysis, and correction factors for scatter in the mass-observable relation were applied to the best-fit masses. The findings for $M_{*} / M_{\text {halo }}$ in this work were consistent with those from RCS for stellar masses above $10^{10} M_{\odot}$. Below that stellar mass, early and late type galaxies were found to have statistically consistent conversion efficiencies (Eq. 3.1), suggesting that stellar mass is a good tracer of halo mass below $M_{*} \sim 10^{10} M_{\odot}$. For a galaxy sample around $L_{*}$, the halo model analysis of the lensing results suggests $M_{\text {halo }} / L=79_{-24}^{+27}$ and $41_{-17}^{+16} M_{\odot} / L_{\odot}(2 \sigma)$ for early and late types, respectively, with higher values at higher luminosity; results at lower luminosity are too noisy to draw conclusions about trends in that direction. 
Using data from the HST GEMS survey, Heymans et al. (2006) analyzed the lensing signal from high stellar mass galaxies over a long redshift baseline, $0.2<z<0.8$. Given the stellar mass limit $\log \left(M_{*} / M_{\odot}\right)>10.5$, the majority of the galaxies exhibit early type morphology; however, no explicit morphological split was used. After modeling the signals using a pure NFW profile without modeling of signal due to satellites or halo vs. stellar mass scatter, they find an average conversion efficiency of $\eta=0.10 \pm 0.03(1 \sigma)$ for the entire sample. These results are consistent with those from lower redshift (Hoekstra et al. 2005; Mandelbaum et al. 2006) when comparing with samples that have a similar stellar mass range. When splitting into redshift bins within the GEMS sample, they find no statistically significant evolution of the $M_{\text {halo }} / M_{*}$ ratio, though their best-fit relation includes slight evolution in the direction of higher ratio at higher redshift, giving an upper limit in growth of this ratio of $\lesssim 2.5$ from $z=0.8$ to the present time.

More recently, Leauthaud et al. (2012a) carried out a joint analysis of the galaxygalaxy lensing, galaxy clustering, and abundance of galaxies as a function of stellar mass (without splitting by galaxy type) in the COSMOS survey. While this survey covers a very small area of the sky, its depth provides a long redshift baseline over which to carry out this analysis. This joint analysis used a quite complex formulation of the halo model with many parameters including ones regulating the scatter between the halo and stellar mass, as described in Leauthaud et al. (2011). For this particular dataset, the stellar mass function has the highest $S / N$ and therefore dominates the constraints on the halo vs. stellar mass relation, which is provided as a functional form with four parameters determined in three redshift bins. The redshift evolution of this halo vs. stellar mass relation is indicative of downsizing, with the stellar mass at which the star baryon conversion efficiency is maximized decreasing at lower redshifts. One new aspect to this halo model analysis compared to previous ones used for lensing analyses is that it provides constraints on the logarithmic scatter in the stellar mass at fixed halo mass, which varied in the range $\sigma_{\log M_{*}}=0.2-0.25$ for the three redshift bins.

The halo model formalism used by Leauthaud et al. (2012a) relied on the use of all galaxies at fixed stellar mass to define the abundance. An updated version of the formalism that permits a split into star-forming and passive galaxies was presented and used by Tinker et al. (2013), also on the COSMOS data. The results of that work suggested that for massive galaxies $\left(M_{*} \gtrsim 10^{10.6} M_{\odot}\right)$, star-forming galaxies form stars at a rate that roughly matches the growth of their dark matter halos via accretion from $z=1$ to 0 , whereas for quiescent galaxies, the growth of their dark matter halos outpaces star formation, reducing their apparent baryon conversion efficiency $\eta$. In contrast, for lower mass galaxies, the halo vs. stellar mass relations are similar for passive and star-forming galaxies at $z<1$. These findings are qualitatively consistent with the trends from the SDSS data using lensing alone in Mandelbaum et al. (2006), but the $S / N$ in COSMOS is superior for lower mass galaxies. Moreover, COSMOS enables tests of redshift evolution, and find a cross-over in the red-blue stellar vs. halo mass relation as a function of redshift, which has not been seen in other surveys (either due to insufficient redshift baseline, or different modeling strategies).

van Uitert et al. (2011) used imaging data from the RCS2 together with SDSS spectroscopic redshifts in order to perform a weak lensing study of the halo vs. stellar mass relation, with a similar halo model formalism as that used in Mandelbaum et al. (2006). They find a relatively steep scaling of halo mass with luminosity, $2.2 \pm 0.1$ and $1.8 \pm 0.1$ for red and blue galaxies, respectively $\dagger$, which is inconsistent with that found by Hoekstra

$\dagger$ These numbers differ from those in van Uitert et al. (2011), and come from a correction that was reported in Velander et al. (2014), which used the same halo modeling software. 
et al. (2005) in the RCS; however, the different methods of modeling the lensing signals and different selection criteria used could be responsible for this difference. The results are consistent with those from the SDSS analysis by Mandelbaum et al. (2006) within the errors. When splitting the sample by stellar mass, van Uitert et al. (2011) confirm the findings from the SDSS: below $10^{11} M_{\odot}$, the stellar mass traces halo mass, but above that stellar mass, the halo masses differ for early and late type galaxies.

van den Bosch et al. (2013) presented a 9-parameter conditional luminosity function (CLF) formalism including various input from $N$-body simulations (halo mass function, concentration-mass relation, and others). Cacciato et al. (2014) showed that when fitting the parameters of this model to describe low-redshift SDSS samples, the resulting CLF was able to describe lensing signals for higher-luminosity and redshift SDSS and RCS2 samples without further adjustment. This result is non-trivially interesting and suggests that the 9-parameter model may be capturing the key features of the galaxy-dark matter connection. One interesting aspect of this model is that it includes constant scatter in $\log L$ at fixed $\log M_{\text {halo }}$, which implies that the scatter in $\log M_{\text {halo }}$ at fixed $\log L$ is not constant (given that the slope of the luminosity-mass relation varies). This aspect of the model is similar to the results of the COSMOS analysis from Leauthaud et al. (2012a), which described the results in terms of a fixed scatter in $\log M_{*}$ at fixed $M_{\text {halo }}$.

Two works (Hudson et al. 2013; Velander et al. 2014) have explored the galaxy-galaxy weak lensing signals as a function of lens galaxy properties using CFHTLenS data. The CFHTLenS data cover sufficient area with enough depth that, compared to the SDSS, RCS, and RCS2, the statistical errors in the lensing signal at lower stellar mass and luminosity have decreased significantly $\dagger$. When fitting the halo mass to a power law in luminosity (stellar mass), Velander et al. (2014) find a power law index of $1.32 \pm 0.06$ and $1.09_{-0.13}^{+0.20}\left(1.36_{-0.07}^{+0.06}\right.$ and $\left.0.98_{-0.07}^{+0.08}\right)$ for red and blue galaxies, respectively. This is shallower than the RCS2 results, possibly because of multiple differences in galaxy selection, as discussed in detail in Velander et al. (2014); however, their results are largely in agreement with the results from the SDSS. Comparison with COSMOS results is somewhat more complex, given that the best-fitting stellar vs. halo mass relationship can only be turned into an average halo mass within each bin when considering the scatter in stellar mass at fixed halo mass. We will return to this point shortly.

Han et al. (2014) carried out a maximum-likelihood weak lensing analysis of opticallyselected galaxy groups in the Galaxy and Mass Assembly (GAMA) survey, using SDSS galaxies as background sources. While the focus of the paper is on groups of galaxies, results are also shown for a set of "groups" with $N_{\text {gal }}=1$, i.e., individual galaxies split by stellar mass (not type or morphology). The trends for the average halo mass as a function of stellar mass were largely consistent with those from previous surveys. One point that was highlighted in both Leauthaud et al. (2011) and Han et al. (2014), and that is quite relevant to the comparison of results from e.g. Velander et al. (2014) against those from Leauthaud et al. (2012a), is that the average halo mass at fixed stellar mass is very sensitive to the lognormal dispersion in $M_{*}$ at fixed $M_{\text {halo }}$ (even more so than on the median $M_{*}$ at fixed $M_{\text {halo }}$ ). Thus, comparison of results of contraining $M_{*}$ as a function of $M_{\text {halo }}$ against measurements of $\left\langle M_{\text {halo }}\right\rangle$ at fixed $M_{*}$ is highly non-trivial. For the GAMA galaxies, Han et al. (2014) found $\sigma_{\log M_{*}} \sim 0.15$, consistent with the COSMOS results within the errors. However, since $\sigma_{\log M_{*}}$ includes not just intrinsic scatter but also measurement error (which could depend on how the stellar mass are estimated and

$\dagger$ The errors are similar to those in COSMOS at low stellar mass, however in the case of the COSMOS analysis, the clustering and stellar mass function plays a significant role in the halo model constraints at low stellar mass. 


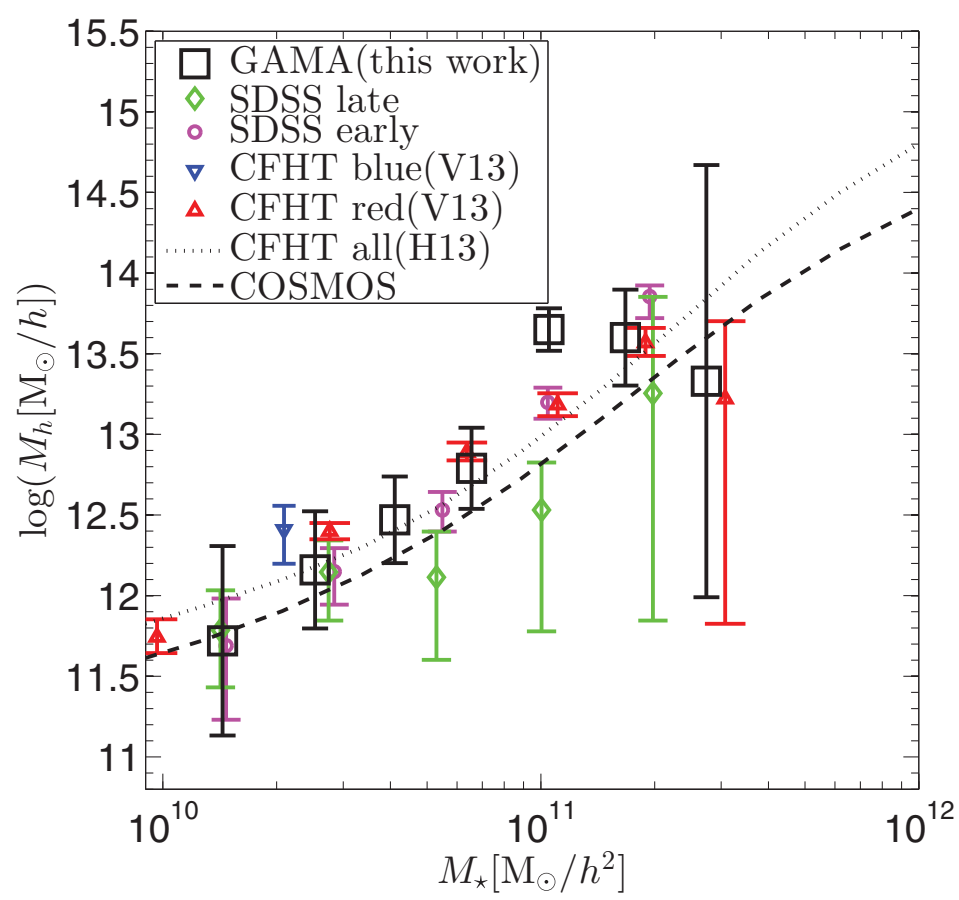

Figure 1. The central halo mass-stellar mass relation measured from weak lensing, from several works described in this section. The quantity that is plotted is the average logarithmic halo mass at fixed stellar mass. The COSMOS measurement, originally provided as a fitted $\left\langle\log \left(M_{\star}\right) \mid M_{h}\right\rangle$ relation, was converted to the plotted quantity by taking into account the scatter in mean stellar mass at fixed halo mass. The same conversion is done for the results from Hudson et al. (2013). It is important to keep in mind that $\left\langle M_{\mathrm{halo}}\right\rangle\left(M_{*}\right)$ depends not just on the mean relationship between these quantities, but also on both their intrinsic scatter and the measurement error in the $M_{*}$. For example, surveys with stellar masses based purely on photometric redshifts, which are an additional source of non-negligible scatter, should have lower average halo masses at fixed stellar mass. Figure used with permission from Han et al. (2014).

whether the dataset has spectroscopic or photometric redshifts), it is not necessarily the case that we even expect similar results from all surveys.

To summarize, in Fig. 1 we show figure 9 from Han et al. (2014), which compares the results for the average halo mass in stellar mass bins (averaged over all galaxy types) from several different papers.

\section{Future challenges}

Aside from the basic observational challenges in weak lensing measurements, which are summarized in e.g. Weinberg et al. (2013), here I discuss some of the challenges in how to make and interpret weak lensing measurements of dark matter halo masses.

The first issue is in defining the observational quantity to be used as a mass tracer, typically either stellar mass and luminosity. For galaxy samples with spectra, the presence of a redshift can be useful in making a robust estimate of these quantities by fitting the SED to templates. However, for lens samples with imaging data alone, photometric redshifts uncertainty becomes uncertainty in the stellar mass and luminosity. The process of carrying out these fits to interpret the SED in terms of stellar mass and luminosity has other sources of uncertainty. The most commonly considered uncertainty is due to the 
stellar initial mass function (IMF), which can lead to tens of percent differences in stellar mass estimates. However, even controlling for IMF differences, Leauthaud et al. (2012b) argue that differences in stellar mass estimates due to the stellar population synthesis model, form chosen for star formation history, and ways of handling dust attenuation can lead to $\sim 45 \%$ uncertainties in the stellar mass estimates. Finally, in the estimate of stellar mass from the mass-to-light ratio and luminosity, we cannot ignore uncertainties that may arise due to the flux estimate that goes into luminosity. This estimate often involves fitting the surface brightness profile of the galaxies, which itself has many pitfalls in the choice of models to use (e.g., Bernardi et al. 2014) and systematic errors due to sky level misestimates near bright foregrounds. These sources of uncertainty make comparison between measurements from different surveys with different analysis techniques necessarily difficult, and in future, we will need to control for such differences quite carefully.

Another issue, which is relevant for studies that attempt to define type-dependent relationships between stellar and halo mass, relates to how the galaxies were split into different types. Morphological estimators can be noisy and difficult to apply to limitedresolution imaging from the ground. Estimators based on spectra, such as the strength of the $4000 \AA$ break, are limited to the special cases where spectra for lens objects are available. Use of rest-frame colors from imaging data can couple photometric redshift errors to the quantity used for type classification, and can also be fooled by dust extinction in edge-on spirals. It is therefore not apparent to what degree we can compare typedependent relationships that are derived in different ways. It is interesting that Hudson et al. (2013) and Velander et al. (2014) found using CFHTLenS that for certain stellar mass bins, their estimates of the halo vs. stellar mass relation differ for certain galaxy types. Naively, since they are using the same source catalog and photometric redshifts, one would expect the noise in their measurements to be correlated, which should result in their agreeing at better than the $1 \sigma$ level. However, the two works differ in their choice of type separator (color vs. spectral type, respectively) and their way of binning the galaxies by stellar mass (directly vs. using luminosity and then getting an average stellar mass for each luminosity bin). They also have some differences in how the mass modeling of the lensing signals was carried out. Differences in how the data were split into samples and modeled must be responsible for the difference between these results.

The final challenges have to do with how halo mass is modeled. The choice of whether to use isolated galaxies (which do not require halo modeling) or not could lead to the selection of unfair samples compared to use of all galaxies regardless of environment. At the same time, the halo model that can be used to interpret the lensing signal for a sample of mixed central and satellite galaxies has its own limitations: for example, it does not include assembly bias (wherein galaxy properties depend not just on halo mass but some other parameter like age; see Zentner et al. 2014 regarding its effects on halo model analyses, and Hearin et al. 2014 for an analysis that attempts to account for assembly bias). It also relies on $N$-body based quantities like the halo mass function and concentration vs. mass relation, which means that if baryonic effects are important (Duffy et al. 2010; Cui et al. 2014) then the halo model predictions will be incorrect and therefore so will the estimated masses. Another factor that is relevant to the studies that already use halo models is the choice of how much complexity to allow. For example, Mandelbaum et al. (2006), van Uitert et al. (2011), and Velander et al. (2014) all used simple halo models with many parameters fixed and with simple corrections for halo mass vs. observable scatter; Leauthaud et al. (2011) and Tinker et al. (2013) used a much more complex halo model with more freedom and fewer fixed parameters, but they also needed to include much more data (stellar mass function and clustering) in order to get strong parameter constraints, which means that systematic uncertainties in 
abundances become more relevant than in the lensing-only studies. It is clear that some of these nuisance parameters are important, and in order to compare results we must understand the impact of choices about which nuisance parameters are held fixed and which are free.

In short, the above complications can complicate a comparison between the different measurements described in Section 3. In the near future, larger lensing surveys are going to make even higher $S / N$ measurements of galaxy-galaxy lensing, which could lead to a better understanding of the halo vs. dark matter connection from weak lensing. At the same time, with the better data will come a need for greater understanding of how these challenges are affecting the results.

\section{Conclusions}

Because of its sensitivity to all types of matter (stars, gas, and dark matter) and its insensitivity to their dynamical state, weak gravitational lensing has emerged as a powerful tool to study the connection between galaxies and their host dark matter halos in the past decade, with results from multiple surveys, the most recent of which have achieved statistical errors in the $5 \%$ regime. While there are a number of challenges to understand in the modeling that is used to go from measured lensing signals to constraints on masses, as described in $\S 4$, there is every reason to believe that near-term surveys like Hyper Suprime-Cam (HSC†, Miyazaki et al. 2006), the Dark Energy Survey (DES $\ddagger$, The Dark Energy Survey Collaboration 2005), and the KIlo-Degree Survey (KIDS $\uparrow$ ) will continue to improve on what we have already learned, and we will learn yet more from the even more ambitious programs that are planned for the coming decade, including the Large Synoptic Survey Telescope (LSST\|, LSST Science Collaborations et al. 2009), Euclid $\dagger \dagger$ (Laureijs et al. 2011), and the Wide-Field Infrared Survey Telescope (WFIRSTAFTA project拉, Spergel et al. 2013).

\section{Acknowledgements}

I am grateful for the support of the Alfred P. Sloan Foundation, which supported my work on this review. I would like to thank Jiaxin Han for permitting me to use figure 9 from his paper, and Alexie Leauthaud and Ying $\mathrm{Zu}$ for useful discussions.

\section{References}

Bernardi, M., Meert, A., Vikram, V., Huertas-Company, M., Mei, S., Shankar, F., \& Sheth, R. K., 2014, MNRAS, 443, 874

Cacciato, M., van Uitert, E., \& Hoekstra, H., 2014, MNRAS, 437, 377

Cooray, A. \& Sheth, R., 2002, Phys. Rep., 372, 1

Cui, W., Borgani, S., \& Murante, G., 2014, MNRAS, 441, 1769

Duffy, A. R., Schaye, J., Kay, S. T., Dalla Vecchia, C., Battye, R. A., \& Booth, C. M., 2010, MNRAS, 405, 2161

Dutton, A. A., Conroy, C., van den Bosch, F. C., Prada, F., \& More, S., 2010, MNRAS, 407, 2

Erben, T., Hildebrandt, H., Miller, L., van Waerbeke, L., Heymans, C., Hoekstra, H., Kitching,

\footnotetext{
$\dagger$ http://www.naoj.org/Projects/HSC/index.html

$\ddagger$ https://www.darkenergysurvey.org/

I http://www.astro-wise.org/projects/KIDS/

|| http://www.lsst.org/lsst

$\dagger \dagger$ http://sci.esa.int/euclid, http://www.euclid-ec.org

$\ddagger \dagger$ http://wfirst.gsfc.nasa.gov/
} 
T. D., Mellier, Y., Benjamin, J., Blake, C., Bonnett, C., Cordes, O., Coupon, J., Fu, L., Gavazzi, R., Gillis, B., Grocutt, E., Gwyn, S. D. J., Holhjem, K., Hudson, M. J., Kilbinger, M., Kuijken, K., Milkeraitis, M., Rowe, B. T. P., Schrabback, T., Semboloni, E., Simon, P.,

Smit, M., Toader, O., Vafaei, S., van Uitert, E., \& Velander, M., 2013, MNRAS, 433, 2545

Gavazzi, R., Treu, T., Rhodes, J. D., Koopmans, L. V. E., Bolton, A. S., Burles, S., Massey, R. J., \& Moustakas, L. A., 2007, ApJ, 667, 176

Gilbank, D. G., Gladders, M. D., Yee, H. K. C., \& Hsieh, B. C., 2011, AJ, 141, 94

Gladders, M. D. \& Yee, H. K. C., 2005, ApJS, 157, 1

Guzik, J. \& Seljak, U., 2001, MNRAS, 321, 439

Han, J., Eke, V. R., Frenk, C. S., Mandelbaum, R., Norberg, P., Schneider, M. D., Peacock, J. A., Jing, Y., Baldry, I., Bland-Hawthorn, J., Brough, S., Brown, M. J. I., Liske, J., Loveday, J., \& Robotham, A. S. G., 2014, preprint (arXiv:1404.6828)

Hearin, A. P., Watson, D. F., Becker, M. R., Reyes, R., Berlind, A. A., \& Zentner, A. R., 2014, MNRAS, 444, 729

Heymans, C., Bell, E. F., Rix, H.-W., Barden, M., Borch, A., Caldwell, J. A. R., McIntosh, D. H., Meisenheimer, K., Peng, C. Y., Wolf, C., Beckwith, S. V. W., Hä,uß,ler B., Jahnke, K., Jogee, S., Sá,nchez S. F., Somerville, R., \& Wisotzki, L., 2006, MNRAS, 371, L60

Heymans, C., Van Waerbeke, L., Miller, L., Erben, T., Hildebrandt, H., Hoekstra, H., Kitching, T. D., Mellier, Y., Simon, P., Bonnett, C., Coupon, J., Fu, L., Harnois Dé,raps J., Hudson, M. J., Kilbinger, M., Kuijken, K., Rowe, B., Schrabback, T., Semboloni, E., van Uitert, E., Vafaei, S., \& Velander, M., 2012, MNRAS, 427, 146

Hoekstra, H., Bartelmann, M., Dahle, H., Israel, H., Limousin, M., \& Meneghetti, M., 2013, Space Sci. Rev., 177, 75

Hoekstra, H., Hsieh, B. C., Yee, H. K. C., Lin, H., \& Gladders, M. D., 2005, ApJ, 635, 73

Hoekstra, H. \& Jain, B., 2008, Annual Review of Nuclear and Particle Science, 58, 99

Hudson, M. J., Gillis, B. R., Coupon, J., Hildebrandt, H., Erben, T., Heymans, C., Hoekstra, H., Kitching, T. D., Mellier, Y., Miller, L., Van Waerbeke, L., Bonnett, C., Fu, L., Kuijken, K., Rowe, B., Schrabback, T., Semboloni, E., van Uitert, E., \& Velander, M., 2013, preprint (arXiv:1310.6784)

Koekemoer, A. M., Aussel, H., Calzetti, D., Capak, P., Giavalisco, M., Kneib, J., Leauthaud, A., Le Fèvre, O., McCracken, H. J., Massey, R., Mobasher, B., Rhodes, J., Scoville, N., \& Shopbell, P. L., 2007, ApJS, 172, 196

Laureijs, R., Amiaux, J., Arduini, S., Auguè,res J. ., Brinchmann, J., Cole, R., Cropper, M., Dabin, C., Duvet, L., \& Ealet, A., et al. 2011, preprint (arXiv:1110.3193)

Leauthaud, A., Tinker, J., Behroozi, P. S., Busha, M. T., \& Wechsler, R. H., 2011, ApJ, 738,45

Leauthaud, A., Tinker, J., Bundy, K., Behroozi, P. S., Massey, R., Rhodes, J., George, M. R., Kneib, J.-P., Benson, A., Wechsler, R. H., Busha, M. T., Capak, P., Cortês, M., Ilbert, O., Koekemoer, A. M., Le Fèvre, O., Lilly, S., McCracken, H. J., Salvato, M., Schrabback, T., Scoville, N., Smith, T., \& Taylor, J. E., 2012a, ApJ, 744, 159

Leauthaud, A., George, M. R., Behroozi, P. S., Bundy, K., Tinker, J., Wechsler, R. H., Conroy, C., Finoguenov, A., \& Tanaka, M., 2012b, ApJ, 746, 95

LSST Science Collaborations, Abell, P. A., Allison, J., Anderson, S. F., Andrew, J. R., Angel, J. R. P., Armus, L., Arnett, D., Asztalos, S. J., \& Axelrod, T. S., et al. 2009, preprint (arXiv:0912.0201)

Mandelbaum, R., Li, C., Kauffmann, G., \& White, S. D. M., 2009, MNRAS, 393, 377

Mandelbaum, R., Seljak, U., \& Hirata, C. M., 2008, J. Cosmology Astropart. Phys., 8, 6

Mandelbaum, R., Seljak, U., Kauffmann, G., Hirata, C. M., \& Brinkmann, J., 2006, MNRAS, 368,715

Mandelbaum, R., Tasitsiomi, A., Seljak, U., Kravtsov, A. V., \& Wechsler, R. H., 2005, MNRAS, 362,1451

Miyazaki, S., Komiyama, Y., Nakaya, H., Doi, Y., Furusawa, H., Gillingham, P., Kamata, Y., Takeshi, K., \& Nariai, K., 2006, in Society of Photo-Optical Instrumentation Engineers (SPIE) Conference Series Vol. 6269 of Society of Photo-Optical Instrumentation Engineers (SPIE) Conference Series, HyperSuprime: project overview

Navarro, J. F., Frenk, C. S., \& White, S. D. M., 1996, ApJ, 462, 563

Reyes, R., Mandelbaum, R., Gunn, J. E., Nakajima, R., Seljak, U., \& Hirata, C. M., 2012, MNRAS, 425, 2610 
Schulz, A. E., Mandelbaum, R., \& Padmanabhan, N., 2010, MNRAS, 408, 1463

Scoville, N., Abraham, R. G., Aussel, H., Barnes, J. E., Benson, A., Blain, A. W., Calzetti, D., Comastri, A., Capak, P., Carilli, C., Carlstrom, J. E., Carollo, C. M., Colbert, J., Daddi, E., Ellis, R. S., Elvis, M., Ewald, S. P., Fall, M., Franceschini, A., Giavalisco, M., Green, W., Griffiths, R. E., Guzzo, L., Hasinger, G., Impey, C., Kneib, J., Koda, J., Koekemoer, A., Lefevre, O., Lilly, S., Liu, C. T., McCracken, H. J., Massey, R., Mellier, Y., Miyazaki, S., Mobasher, B., Mould, J., Norman, C., Refregier, A., Renzini, A., Rhodes, J., Rich, M., Sanders, D. B., Schiminovich, D., Schinnerer, E., Scodeggio, M., Sheth, K., Shopbell, P. L., Taniguchi, Y., Tyson, N. D., Urry, C. M., Van Waerbeke, L., Vettolani, P., White, S. D. M., \& Yan, L., 2007a, ApJS, 172, 38

Scoville, N., Aussel, H., Brusa, M., Capak, P., Carollo, C. M., Elvis, M., Giavalisco, M., Guzzo, L., Hasinger, G., Impey, C., Kneib, J., LeFevre, O., Lilly, S. J., Mobasher, B., Renzini, A., Rich, R. M., Sanders, D. B., Schinnerer, E., Schminovich, D., Shopbell, P., Taniguchi, Y., \& Tyson, N. D., 2007b, ApJS, 172, 1

Seljak, U., 2000, MNRAS, 318, 203

Spergel, D., Gehrels, N., Breckinridge, J., Donahue, M., Dressler, A., Gaudi, B. S., Greene, T., Guyon, O., Hirata, C., Kalirai, J., Kasdin, N. J., Moos, W., Perlmutter, S., Postman, M., Rauscher, B., Rhodes, J., Wang, Y., Weinberg, D., Centrella, J., Traub, W., Baltay, C., Colbert, J., Bennett, D., Kiessling, A., Macintosh, B., Merten, J., Mortonson, M., Penny, M., Rozo, E., Savransky, D., Stapelfeldt, K., Zu, Y., Baker, C., Cheng, E., Content, D., Dooley, J., Foote, M., Goullioud, R., Grady, K., Jackson, C., Kruk, J., Levine, M., Melton, M., Peddie, C., Ruffa, J., \& Shaklan, S., 2013, preprint (arXiv:1305.5422)

The Dark Energy Survey Collaboration, 2005, ArXiv Astrophysics e-prints

Tinker, J. L., Leauthaud, A., Bundy, K., George, M. R., Behroozi, P., Massey, R., Rhodes, J., \& Wechsler, R. H., 2013, ApJ, 778, 93

van den Bosch, F. C., More, S., Cacciato, M., Mo, H., \& Yang, X., 2013, MNRAS, 430, 725

van Uitert, E., Hoekstra, H., Velander, M., Gilbank, D. G., Gladders, M. D., \& Yee, H. K. C., 2011, A\& $A, 534, \mathrm{~A} 14$

Velander, M., van Uitert, E., Hoekstra, H., Coupon, J., Erben, T., Heymans, C., Hildebrandt, H., Kitching, T. D., Mellier, Y., Miller, L., Van Waerbeke, L., Bonnett, C., Fu, L., Giodini, S., Hudson, M. J., Kuijken, K., Rowe, B., Schrabback, T., \& Semboloni, E., 2014, MNRAS, 437,2111

Weinberg, D. H., Mortonson, M. J., Eisenstein, D. J., Hirata, C., Riess, A. G., \& Rozo, E., 2013, Phys. Rep., 530, 87

York, D. G., Adelman, J., Anderson, Jr. J. E., Anderson, S. F., Annis, J., Bahcall, N. A., Bakken, J. A., Barkhouser, R., Bastian, S., Berman, E., Boroski, W. N., Bracker, S., Briegel, C., Briggs, J. W., Brinkmann, J., Brunner, R., Burles, S., Carey, L., Carr, M. A., Castander, F. J., Chen, B., Colestock, P. L., Connolly, A. J., Crocker, J. H., Csabai, I., Czarapata, P. C., Davis, J. E., Doi, M., Dombeck, T., Eisenstein, D., Ellman, N., Elms, B. R., Evans, M. L., Fan, X., Federwitz, G. R., Fiscelli, L., Friedman, S., Frieman, J. A., Fukugita, M., Gillespie, B., Gunn, J. E., Gurbani, V. K., de Haas E., Haldeman, M., Harris, F. H., Hayes, J., Heckman, T. M., Hennessy, G. S., Hindsley, R. B., Holm, S., Holmgren, D. J., Huang, C.-h., Hull, C., Husby, D., Ichikawa, S.-I., Ichikawa, T., Ivezić, Ž,., Kent, S., Kim, R. S. J., Kinney, E., Klaene, M., Kleinman, A. N., Kleinman, S., Knapp, G. R., Korienek, J., Kron, R. G., Kunszt, P. Z., Lamb, D. Q., Lee, B., Leger, R. F., Limmongkol, S., Lindenmeyer, C., Long, D. C., Loomis, C., Loveday, J., Lucinio, R., Lupton, R. H., MacKinnon, B., Mannery, E. J., Mantsch, P. M., Margon, B., McGehee, P., McKay, T. A., Meiksin, A., Merelli, A., Monet, D. G., Munn, J. A., Narayanan, V. K., Nash, T., Neilsen, E., Neswold, R., Newberg, H. J., Nichol, R. C., Nicinski, T., Nonino, M., Okada, N., Okamura, S., Ostriker, J. P., Owen, R., Pauls, A. G., Peoples, J., Peterson, R. L., Petravick, D., Pier, J. R., Pope, A., Pordes, R., Prosapio, A., Rechenmacher, R., Quinn, T. R., Richards, G. T., Richmond, M. W., Rivetta, C. H., Rockosi, C. M., Ruthmansdorfer, K., Sandford, D., Schlegel, D. J., Schneider, D. P., Sekiguchi, M., Sergey, G., Shimasaku, K., Siegmund, W. A., Smee, S., Smith, J. A., Snedden, S., Stone, R., Stoughton, C., Strauss, M. A., Stubbs, C., SubbaRao, M., Szalay, A. S., Szapudi, I., Szokoly, G. P., Thakar, A. R., Tremonti, C., Tucker, D. L., Uomoto, A., Vanden Berk, D., Vogeley, M. S., Waddell, P., Wang, S.-i., Watanabe, M., Weinberg, D. H., Yanny, B., Yasuda, N., \& SDSS Collaboration 2000, AJ, 120, 1579

Zentner, A. R., Hearin, A. P., \& van den Bosch, F. C., 2014, MNRAS, 443, 3044 